ORIGINAL PAPER

\title{
EVALUATION OF PATIENTS WITH PATHOLOGY AT RISK FOR FOREARM AND HAND AMPUTATION
}

\author{
M. K. Pogărășteanu ${ }^{1,2}$, A. Ioncu ${ }^{3}$, M. Moga ${ }^{1,2}$, Gabriela Soare ${ }^{1,4}$, R. Adam ${ }^{5}$, A. G. Barbilian ${ }^{1,2}$ \\ 1"Carol Davila" University of Medicine and Pharmacy, Bucharest, Romania \\ 2“Dr. Carol Davila" Central University Emergency Military Hospital, Bucharest, Romania \\ ${ }^{3}$ Centre Hospitalier de la Dracénie, Draguignan, France \\ ${ }^{4}$ Bucharest Emergency Clinical Hospital, Bucharest, Romania \\ 5"Elias" Emergency University Hospital, Bucharest, Romania
}

Corresponding author: Mark Pogărășteanu

Email: mark.pogarasteanu@gmail.com

\begin{abstract}
Forearm and hand pathologies, such as trauma, infections, tumors or extensive burns, represent a genuine challenge for the orthopedic surgeon, considering the fact that they have a major impact on the patient quality of life. Amputations at this level, though rare, are often a lifesaving procedure, which helps minimize the severe complications that may occur. We present a retrospective study made on a number of 3203 patients which were admitted to an Orthopedic clinic, from 2012 to 2014. We analyzed which is the most frequent cause for upper limb procedures, the distribution in both genders, the average hospitalization period of time and we determined which is the higher occurrence between lower and upper limb pathologies, aiming to determine the prevalence of forearm and hand amputations in the studied group.
\end{abstract}

KEYWORDS: forearm, hand, amputation

\section{INTRODUCTION}

In most case, equipping the upper limb with an external prosthesis is equivalent with using a forearm and hand prosthesis, as these are the two areas most often subjected accidents that lead to an amputation $[1,2]$. This is due to the involvement of the forearm and hand in heavy labor, that often results in accidents, especially in patients of male sex, aged between 20 and 40 years old $[3,4,5]$. Another pathology that may lead to an amputation of the forearm and of the hand are tumors, both as a primary location as well as metastatic; the primary tumors may be malignant in nature or aggressively benign $[6,7]$. Besides the situations mentioned before, the forearm and hand amputation also have indication in infections, extensive burns, frostbites and irreversible segment ischemia.

One aspect that remains inconclusive is in which extent and in what way does a group of patients that were treated surgically in an Orthopedics Clinic at the level of the upper limb relate to patients that were treated surgically at the forearm and hand, and in what manner does the pathology, be it traumatic [8], tumoral or infectious in nature, lead to the necessity of a surgical amputation, in the context in which there are, at least theoretically, a wide array of procedures that may prevent a forearm or hand amputation. 
The necessity of exploring this relationship comes from the reality of the fact that the surgical indication determines the use of one or another surgical technique; thus an amputation for trauma in emergency will need a classic technique, while an amputation that may be planned will offer the possibility of choosing preoperatively a certain type of exoprosthesis, which in turn will need a certain variant of the established techniques, such as an osteomyoplastic or an CONM procedure (circumferential osteoneuromyoplastic) [9].

Moreover, patients that undergo a surgical procedure, including amputation of the upper limb, have comorbidities, and these need to be evaluated and treated before surgery if warranted.

\section{MATERIALS AND METHOD}

The main objective of this study is to evaluate the traumatic, tumoral and infectious pathologies of the forearm and hand in the group of patients treated surgically in the Orthopedics Clinic of the Centre Hospitalier de la Dracénie Hospital, Draguignan, France, from 2012 until 2014. We aim to evaluate the number of patients, the ratio of male to female patients, their age, as well as the number of hospitalization days, in comparison to the group of patients that suffered an intervention at the level of the upper limb, in the same clinic and in the same period, in order to determine if there are any significant differences. We aim to determine whether there are cases of amputation at the level of the forearm and of the hand in the given patient group, and to evaluate these cases using the same criteria.

Our first hypothesis is that the cases of amputation at the level of the forearm and of the hand represent a very small percentage of the overall orthopedic procedures in the studied group. Our second hypothesis is that the groups of patients with traumatic, tumoral respectively infectious pathologies at the level of the forearm and of the hand have different characteristics compared to the groups of patients with the same pathologies at the level of the thoracic limb as a whole and compared to the whole analyzed group of patients.

In order to achieve the objectives and to test our hypothesis we developed a retrospective observational study in which we analyzed a database from 2012 to 2014, holding statistical data of the patients that were admitted to the Orthopedic Clinic of the Centre Hospitalier de la Dracénie Hospital, Draguignan, France. We gained access to this data following a CERO $\mathrm{PhD}$ scholarship of the main author in the aforementioned Clinic, from 20.05.2015 to 20.07.2015, in accordance with the officially approved research plan.

The patients were selected according to a series of inclusion and exclusion criteria.

Inclusion criteria: both male and female patients that were admitted and treated surgically in the Orthopedic Clinic Centre Hospitalier de la Dracénie Hospital, Draguignan, France from 2012 to 2014, aged 1 to 110 years old.

Exclusion criteria: patients with incomplete data records; patients that were consulted but not treated surgically.

Data regarding comorbidities were not collected, even though the associated pathologies are relevant and ought to be evaluated thoroughly, as we believe this data to go beyond the purpose of this study.

The database initially held 31102 lines of data organized in an Excel spreadsheet, each line of data representing one patient and divided in 24 columns defining statistic, anthropometric, diagnostic and interventional data. The statistical data included: record number (NUM SEJOUR), date of admittance to the hospital unit (DATE ENTREE SEJ), date of discharge from the hospital unit (DATE SORTIE SEJ), duration of hospitalization (DUREE RSS), ID code for the period of hospitalization (ID SEJOUR), date of admittance to the Orthopedic Clinic (DATE DEBUT MVT), date of discharge from the Orthopedic Clinic (DATE FIN MVT), duration of stay in the Orthopedic Clinic (DUREE MVT), ID code of the stay in the Orthopedic Clinic (IDENTIFIANT MVT), number of inter-clinic transfers (NB RUM DANS RSS), type of admittance (TYPE SEJOUR), unit code (CODE UNITEE RUM) and the year of discharge (ANEE SORTIE SEJ). The anthropometric data included: the patient's age at admittance (AGE PATIENT ANNEE ENT SEJ) and the patient's sex (SEXE). The diagnostic data included: the type of diagnosis (TYPE DIAG), the code of the main retained diagnosis (DP RETENU), the code of diagnosis (CODE DIAG) and the standard formulated diagnosis (LIBELLE DIAG). 
The interventional data included: the procedure code (CODE ACTE), the standardized procedure name (LIBELLE ACTE), the classification of the procedure (CLASSANT), the activity code (CODE ACTIVITE) and the individual ID code of the procedure (ID ACTE).

In the first stage we eliminated all the secondary diagnosis, so that the database was left with a total of 3483 lines of data, each corresponding to a main diagnosis. We also eliminated all the columns that were considered to have no significance to the study: DATE ENTREE SEJ, DATE SORTIE SEJ, TYPE SEJOUR, CODE UNITEE RUM, ANEE SORTIE SEJ, CODE DIAG, TYPE DIAG, CLASSANT, CODE ACTIVITE, IDENTIFIANT MVT, ID SEJOUR, ID ACTE, NB RUM DANS RSS.

In the second stage we identified the cases in which the same patient, identified by the individual admittance ID number, was subjected to a minimum of two surgical procedures during the same hospital stay; this allowed us to determine the total number of patients included in the study, which is 3203 .

In a third stage we extracted the data that refers to the general characteristics of the patients: their number, sex, age (minimum, maximum, average), as well as the duration of hospital stay. We summarized the data regarding the surgical procedures on the thoracic limb and on the forearm and the hand, in the given time periods, according to the previously mentioned indicators. We determined and compared the number of patients to the number of surgical procedures performed, for each year, in the overall group including all orthopedic pathologies concerning the upper limb. We determined and compared the number of patients of masculine sex versus feminine sex, as well as the minimum, average and maximum ages and the durations of hospital stay for 2012, 2013 and 2014 in the given patient groups. Regarding the patients that were subjected to surgical procedures at the level of the forearm and of the hand, we analyzed: the number of patients versus the number of surgical procedures, for 2012, 2013 and 2014; the distribution of the two genders for each year; the minimum, average and maximum age for each year and the average duration of hospital stay for each year. This analysis was also performed for traumatic, tumoral and infectious pathologies.

We determined the total number of amputations at the level of the forearm and of the hand, and we interpreted the results.

\section{RESULTS}

The total number of patients was 3203, of which $42 \%$ were presented with a pathology of the upper limb, and 58\% presented a pathology of the lower limb and of the spine, taken as a single group. In total, 3483 surgical procedures were performed. In the studied population we observed that the male sex was represented by 1413 patients, $44 \%$ of the entire population, while the female sex was represented by 1790 patients, 54\% of the entire population, $\mathrm{M}: \mathrm{F}=$ $1: 1.26$. The age of the patients varies between 6 and 101 years, with an average of 61.1 years and a standard deviation of 20.67. The duration of hospital stay for the whole studied population varied from 0 days (interpreted as day surgery, $<24$ hours) and 54 days, values that are identical to those found for the stay in the Orthopedics Clinic, with a different average stay: 5.36 days in the first case (6.41 standard deviation) and 5.04 in the second case (5.94 standard deviation).

The summed-up analysis divided by age of the patients on which a surgical procedure was performed at the level of the upper limb is presented in Table 1.

\begin{tabular}{lcccc}
\hline & $\mathbf{2 0 1 2}$ & $\mathbf{2 0 1 3}$ & $\mathbf{2 0 1 4}$ & Total \\
\hline No. of patients & 396 & 399 & 548 & 1343 \\
\hline $\mathrm{M}$ & 176 & 187 & 295 & 658 \\
\hline $\mathrm{F}$ & 220 & 212 & 253 & 685 \\
\hline Minimum age & 6 & 12 & 11 & 9 \\
\hline Maximum age & 92 & 99 & 100 & 100 \\
\hline Average age & 56.56 & 54.24 & 52.82 & 54.54 \\
\hline $\begin{array}{l}\text { Duration of } \\
\text { hospital stay }\end{array}$ & 1.67 & 1.48 & 1.6 & 1.58 \\
\hline $\begin{array}{l}\text { Number of } \\
\text { procedures }\end{array}$ & 427 & 428 & 644 & 1499 \\
\hline
\end{tabular}

Table 1 - The data for the group that had surgical interventions of the upper limb

The comparative analysis regarding the number of patients versus the number of surgical procedures from 2012 to 2014 in the area of pathology of the upper limb shows a slight increase in 2014 of both the number of patients 
as well as the number of surgical procedures. Comparing the distribution of patients according to their sex from 2012 to 2014 in the same area of pathology shows a higher number of male patients, peaking in September 2013, a tendency that becomes more accentuated in 2014, with an increase in the numerical value between the two genders in favor of the masculine gender. The distribution of patients according to the minimal, average and maximum age for 2012, 2013 and 2014 shows a large interval for the age extremes with a relatively constant age average. For the same period and pathology, the comparative analysis of the average duration of the hospital stay is shown in Figure 1. We see a fluctuant monthly evolution, with the exception of an increase in the hospitalization periods in August and September of 2012 and 2013.

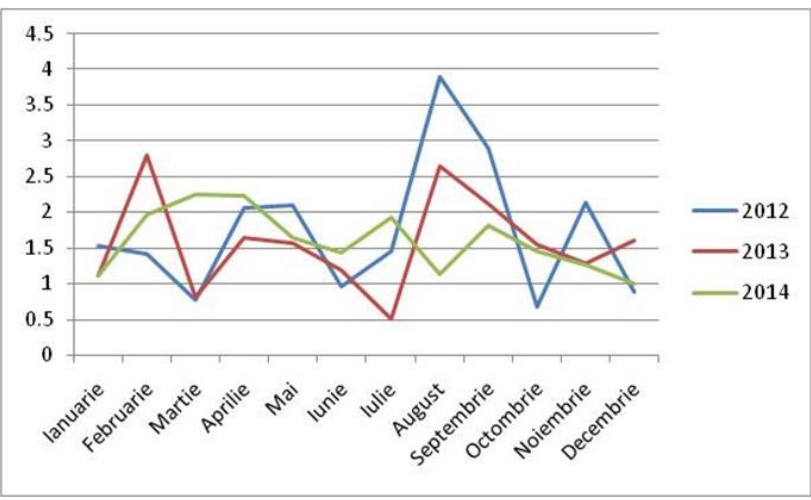

Figure 1 - The average hospital stays in days, for upper limb pathology

The analysis by year of the group of patients that were operated for trauma at the level of the forearm and of the hand between 2012 and 2014 is summarized in Table 2.

\begin{tabular}{lllll}
\hline & $\mathbf{2 0 1 2}$ & $\mathbf{2 0 1 3}$ & $\mathbf{2 0 1 4}$ & Total \\
\hline No. of patients & 120 & 95 & 173 & 388 \\
\hline $\mathrm{M}$ & 38 & 44 & 89 & 171 \\
\hline $\mathrm{F}$ & 82 & 51 & 84 & 217 \\
\hline Minimum age & 14 & 15 & 14 & 14 \\
\hline Maximum age & 92 & 92 & 94 & 94 \\
\hline Average age & 58.8 & 55.73 & 51.79 & 55.44 \\
\hline $\begin{array}{l}\text { Duration of } \\
\text { hospital stay }\end{array}$ & 2.81 & 2.4 & 2.3 & 2.5 \\
\hline $\begin{array}{l}\text { Number of } \\
\text { procedures }\end{array}$ & 130 & 99 & 201 & 430 \\
\hline
\end{tabular}

Table 2 - The summarized data for the group of patients that were operated for trauma at the level of the forearm and of the hand
The analysis by year of the group of patients that were operated for tumors at the level of the forearm and of the hand between 2012 and 2014 is summarized in Table 3.

\begin{tabular}{lllll}
\hline & $\mathbf{2 0 1 2}$ & $\mathbf{2 0 1 3}$ & $\mathbf{2 0 1 4}$ & Total \\
\hline No of patients & 9 & 12 & 17 & 38 \\
\hline $\mathrm{M}$ & 2 & 5 & 11 & 18 \\
\hline $\mathrm{F}$ & 7 & 7 & 6 & 20 \\
\hline Minimum age & 6 & 17 & 11 & 6 \\
\hline $\begin{array}{l}\text { Maximum } \\
\text { age }\end{array}$ & 71 & 76 & 77 & 77 \\
\hline Average age & 43.44 & 37.66 & 43.05 & 41.38 \\
\hline $\begin{array}{l}\text { Duration of } \\
\text { hospital stay }\end{array}$ & 0.11 & 0 & 0.05 & 0.05 \\
\hline $\begin{array}{l}\text { Number of } \\
\text { procedures }\end{array}$ & 9 & 12 & 19 & 40 \\
\hline
\end{tabular}

Table 3 - The summarized data for the group of patients that were operated for tumors at the level of the forearm and of the hand

The analysis by year of the group of patients that were operated for infections at the level of the forearm and of the hand between 2012 and 2014 is summarized in Table 4. We point out that there are only two cases reported.

\begin{tabular}{lllll}
\hline & $\mathbf{2 0 1 2}$ & $\mathbf{2 0 1 3}$ & $\mathbf{2 0 1 4}$ & Total \\
\hline No. of patients & 0 & 0 & 2 & 2 \\
\hline $\mathrm{M}$ & 0 & 0 & 1 & 1 \\
\hline $\mathrm{F}$ & 0 & 0 & 1 & 1 \\
\hline Minimum age & 0 & 0 & 16 & 16 \\
\hline Maximum age & 0 & 0 & 62 & 62 \\
\hline Average age & 0 & 0 & 39 & 39 \\
\hline $\begin{array}{l}\text { Duration of } \\
\text { hospital stay }\end{array}$ & 0 & 0 & 0 & 0 \\
$\begin{array}{l}\text { Number of } \\
\text { procedures }\end{array}$ & 0 & 0 & 2 & 2 \\
\hline
\end{tabular}

Table 4 - The summarized data for the group of patients that were operated for infections at the level of the forearm and of the hand

Table 5 shows the evaluated indicators for trauma, tumors and infections, for all the patients operated at the level of the forearm and of the hand. 


\begin{tabular}{lccc}
\hline & Traumatic & Tumoral & Infectious \\
\hline Number of patients & 388 & 38 & 2 \\
\hline $\mathrm{M}$ & 171 & 18 & 1 \\
\hline $\mathrm{F}$ & 217 & 20 & 1 \\
\hline Minimum age & 14 & 6 & 62 \\
\hline Maximum age & 94 & 77 & 39 \\
\hline Average age & 55.44 & 41.38 & 0 \\
\hline Duration of hospital stay & 2.5 & 0.05 & 2 \\
\hline Number of procedures & 430 & 40 & 2 \\
\hline
\end{tabular}

Table 5 - The total number of patients operated at the level of the forearm and of the hand, from 2012 to 2014

The number of surgical procedures performed at the level of the forearm and of the hand between 2012 and 2014 according to each pathology are shown in Figure 2.

\section{Traumatic $=$ Tumoral $=$ Infectios}

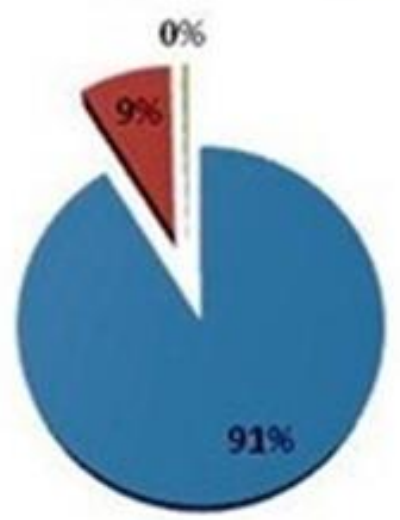

Figure 2 - The total number of procedures performed at the level of the forearm and hand, by percentage

The total number of amputations performed at the level of the forearm and hand, between 2012 and 2014, is one, in November 2012.

\section{DISCUSSION}

Regarding the analysis of the study involving the database of cases of patients onto which a surgical procedure was performed between 2012 and 2014 in the Orthopedics Clinic of the Centre Hospitalier Hospital in Dracénie, Draguignan, France, we note that of the total 3203 patients, $42 \%$ represent the group of patients that have been diagnosed with a pathology of the upper limb, while 58\% represent the patients that have been diagnosed with a pathology of the spine and lower limb, combined. A total of 3483 surgical procedures were performed in these cases, of which approximately $43 \%$ were interventions on the upper limb, and approximately $57 \%$ were interventions on the lower limb and on the spine, combined.

Out of the 1343 patients that suffered surgical interventions on their upper limbs, 428 $(31.8 \%)$ were performed at the level of the forearm and hand, and of the latter, 388 patients $(90.6 \%)$ were operated for trauma, $38(8.87 \%)$ for tumor and $2(0.4 \%)$ for infection.

Out of the 1499 surgical procedures performed on the upper limb, 472 (31.48\%) were performed on the forearm and hand, and of which, $430(91.1 \%)$ were done for trauma, 40 $(8.47 \%)$ for tumor and $2(0.4 \%)$ for infection.

The comparative analysis regarding the number of patients versus the number of surgical procedures performed between 2012 and 2014 for pathologies affecting the upper limb shows a slight increase in 2014 of both the number of patients as well as in the number of performed surgeries, when compared to the previous two years. When we compared the number of admitted patients to the number of performed surgeries for trauma of the forearm and limb between 2012 and 2014, we saw a fluctuating evolution of both the number of cases as well as of the number of surgeries, with a tendency for an increase of the latter during the summer months. Fluctuation that can be noted also in the duration of stay in the hospital unit, with an increase in the months of august and September of 2012 and 2013, most probably related to a higher number of tourists in the area.

Regarding the surgical interventions for infectious pathology at the level of the forearm and of the hand, between 2012 and 2014, we 
must point out that there were only two reported cases.

In the initial group, male patients were $44.11 \%$ of all patients, while female patients were $55.88 \%$. In the group of patients that suffered a surgical procedure at the level of the upper limb, male patients were $48.99 \%$, while female patients were $51.01 \%$. In the group of patients that suffered a surgical procedure at the level of the forearm and hand, those operated for trauma were $44.07 \%$ male and $55.92 \%$ female, those operated for tumors were $47.36 \%$ female and $52.63 \%$ male and those operated for infection were $50 \%$ male and $50 \%$ female. The patients' distribution according to sex, between 2012 and 2014, in the group with a pathology of the upper limb, shows a predominance of male patients, with a peak in September 2013, a tendency that accentuates in 2014, with an increase in the numerical difference between the two sexes, favoring the males. The comparative analysis regarding the distribution of patients according to sex, between 2012 and 2014, shows a greater number of male patients compared to females, as well as an absence of data regarding the month of February 2012.

The age of the patients from the initial group has an overall minimal value of 6 (years), an overall maximum value of 101 (years) and an average of 61.1 (years). The patients that have suffered a surgical procedure for an upper limb pathology have had a minimal age of 6 years old, a maximum age of 100 years and an average of 54.54 years. The patients that have suffered a surgical intervention at the level of the forearm and hand for trauma have had the age interval between 14 and 94 years old, with an average of 55.44 years; for tumors have had the age interval between 6 and 77 years old, with an average of 41.38 years and for infection have had the age interval between 16 and 62 years old, with an average of 39 years.

In the comparative analysis regarding the distribution of patients according to minimum, average and maximum age, between 2012 and 2014, in the group that includes all the pathologies concerning the upper limb, we see a large interval of age, but with a relatively constant age average. We see a slightly fluctuant evolution in the distribution of the patients according to minimum, average and maximum age, between 2012 and 2014, in the group operated for a traumatic pathology of the forearm and hand, with an absence of data for February 2012 and a notable increase of the minimum and average age for June 2012.

Of all the cases that were taken into account in our study, we found just one case where an amputation was performed at the level of the forearm and of the hand, more precisely a finger amputation for a female patient, in 2012, for trauma. This represents $0.21 \%$ of all surgical procedures performed at the level of the forearm and hand, and $0.23 \%$ of all procedures performed for trauma.

We consider that the study has reached its objectives, as we have evaluated the relationship between multiple indicators that characterize the groups of interventions performed for trauma, tumor and infection, both in relation to each other as well as to the rest of the procedures performed in the same period of time; also, we found and characterized the incidence of amputation as a procedure performed at the level of the forearm and of the hand.

\section{CONCLUSION}

By analyzing the data from this retrospective study, we can conclude that, although the pathology that in theory would represent a possible source of indications for amputation of the forearm and/or hand is well represented, an amputation at the mentioned level is a rare occurrence in the given patient sample.

As for the differences between the initial group of patients, the group of patients that were subjected to surgical procedures at the level of the upper limb and those that were subjected to surgical procedures just at the level of the forearm and hand, we may conclude that:

- most surgical procedures of the forearm and hand were performed for trauma;

- there is a higher addressability during the summer months;

- there is a slight predominance of female patients versus males in all groups;

- there is a tendency for an increased lower and average age of patients with a traumatic pathology in the area of the forearm and hand, in comparison to the other groups;

- the patients that were admitted for forearm and hand trauma had a longer period of 
hospitalization compared to the other upper limb groups, but shorter when compared to the patients that were admitted for spine and lower limb pathologies.

\section{REFERENCES}

[1] TR Dillingham, LE Pezzin, EJ MacKenzie, "Limb amputation and limb deficiency: epidemiology and recent trends in the United States", South Med J, 2002, 95(8):875-83

[2] K Ziegler-Graham, EJ MacKenzie, PL Ephraim, TG Travison, R Brookmeyer, "Estimating the prevalence of limb loss in the United States: 2005 to 2050", Arch Phys Med Rehabil, 2008, 89(3):422-29

[3] B Morrey, J Sanchez-Sotelo, "The Elbow and its Disorders", Saunders Elsevier, 2009

[4] R Baumgartner, "The Surgery of Arm and Forearm Amputation", Saunders Elsevier, s1: vol 12, 1981

[5] ST Canale, "Campbell's Operative Orthopaedics 11th Edition", Mosby Elsevier, 2008

[6] SA Rosenberg, H Kent, J Costa, "Prospective Randomized Evaluations of the Role of LimbSparing Surgery, Radiation Therapy and Adjuvant Chemoimmunotherapy in the Treatment of Adult Soft Tissue Sarcoma", Surgery, 1978 Jul, 84(1):62-9

[7] R Bryan, E Soule, J Dobyns, D Pritchard, R Linscheid, "Primary Epithelioid Sarcoma of the Hand and Forearm: a review of Thirteen Cases", J Bone Joint Surg Am, 1974 Apr, 56(3):458-65

[8] S Tintle, M Baechler, G Nanos, J Forsberg, B Potter, "Traumatic and Trauma-Related Amputations, part II: Upper Extremity and Future Directions", J Bone Joint Surg Am, 2010, 92(18):2934-45

[9]https://www.drks.de/drks_web/navigate.do?navig ationId=trial.HTML\&TRIAL_ID=DRKS00004868.

[Online] [Cited: 09 09, 2019.] 